\title{
Chemotherapeutic alteration of VEGF-/PDGF- and PDGF-R $\alpha / \beta$ expression by imatinib in HPV-transformed squamous cell carcinoma compared to HPV-negative HNSCC in vitro
}

\author{
J.D. SCHULTZ ${ }^{1}$, K. MÜHLHEIM ${ }^{1}$, P. ERBEN ${ }^{2}$, R.D. HOFHEINZ ${ }^{2}$, A. FABER ${ }^{1}$, \\ C. THORN ${ }^{1}$, J.U. SOMMER ${ }^{1}$, K. HÖRMANN ${ }^{1}$ and A. SAUTER ${ }^{1}$ \\ Departments of ${ }^{1}$ Otorhinolaryngology, Head and Neck Surgery, and ${ }^{2}$ Hematology and Oncology, \\ Medical Faculty of Mannheim, University of Heidelberg, Mannheim, Germany
}

Received May 9, 2011; Accepted June 6, 2011

DOI: $10.3892 /$ or.2011.1403

\begin{abstract}
Head and neck squamous cell carcinoma (HNSCC) is an aggressive epithelial malignancy known to be the most common neoplasm appearing in the upper aerodigestive tract. The poor five-year survival rate has remained unchanged in the last decades despite the emergence of improved techniques in surgery, radiation and chemotherapy. In the last 20 years awareness of a subset of squamous cell carcinomas induced by oncogenic forms of the human papilloma virus (HPV) (high-risk types 16 and 18) has increased. The incidence of HPV-associated oropharyngeal cancer is rising, indicating the increased importance of the viral etiology. Cell proliferation, migration, induction of tumor vascularization and carcinogenesis, as well as invasion facilitation is regulated by a variety of angiogenic peptides like PDGF, PDGF-R and VEGF. They might be an encouraging target for biological anticancer therapy by inhibiting disrupted cellular signaling pathways. Imatinib has been shown to target specific tyrosine kinases, inhibiting proliferation in various cancer entities. The purpose of this study was
\end{abstract}

Correspondence to: Dr J.D. Schultz, Universitäts HNO-Klinik, Universitätsmedizin Mannheim, Theodor Kutzer Ufer 1-3, 68167 Mannheim, Germany

E-mail: johannes.schultz@umm.de

Abbreviations: ABL, abelson murine leukemia viral oncogene homolog; CAF, cancer-associated fibroblasts; CML, chronic myeloid leukemia; DNA, deoxyribonucleic acid; ECM, extracellular matrix; ELISA, enzyme-linked immunosorbent assay; HNSCC, head and neck squamous cell carcinoma; NSCLC, non-small cell lung carcinoma; p53, tumor protein 53; PBS-buffer, phosphate-buffered saline-buffer; PDGF, platelet-derived growth factor; PDGF-R $\alpha / \beta$, platelet-derived growth factor-receptor $\alpha / \beta$; PTKs, protein tyrosine kinase; SCC, squamous cell carcinoma (cervix uteri); SDS-PAGE, sodium dodecyl sulfate polyacrylamide gel electrophoresis; TGF, transforming growth factor; VEGF, vascular endothelial growth factor

Key words: imatinib, VEGF, PDGF, PDGF-R $\alpha / \beta$, HNSCC, proteintyrosine-kinase, human papilloma virus to evaluate the expression pattern of angiogenic factors (VEGF, PDGF and PDGF-R) in HPV-positive (p16-CERV196 SCC) and (-negative squamous cell carcinoma (HNSCC). The study also evaluated the vulnerability of anti-angiogenesis therapy depending on the HPV status as potential treatment modality compared to established platinum-based chemotherapeutic drugs. The different squamous tumor cell lines were incubated with increasing concentrations of carboplatin ( 3 and $7.5 \mu \mathrm{mol}$ ) and imatinib (18 and $30 \mu \mathrm{mol})$. ELISA immunohistochemical methods were carried out after 48, 72, 120, 192 and $240 \mathrm{~h}$. We demonstrated a significant reduction of VEGF and PDGF-R $\alpha / \beta$ expression patterns after incubation of imatinib in ELISA and immunohistochemical methods, irrespective of the HPV status of the tumor cells, whereas the application of carboplatin had no impact on the expression of angiogenic peptides. Viral oncogen-transformed squamous cell carcinoma (CERV196) cells were characterized by a reduced susceptibility for an imatinib-altered VEGF expression. Further studies are planned to investigate this observance in HPV-positive HNSCC in vitro. The implementation of a selective molecular anti-angiogenic therapy in established chemotherapeutic regimens may enhance the efficacy of platinum-based chemotherapy without an increased toxicity profile and could thus improve the clinical outcome in HNSCC, irrespective of the HPV status.

\section{Introduction}

Head and neck squamous cell carcinoma (HNSCC) is an aggressive epithelial malignancy known to be the most common neoplasm appearing in the upper aerodigestive tract. With a global annual incidence of approximately 644,000 cases and 352,000 associated deaths, HNSCC is the fifth most common cancer worldwide $(1,2)$. Head and neck cancer is a heterogeneous group that differs greatly in tumor aggressiveness and response to treatment. The poor five-year survival rate has remained unchanged in the last decades even though improved techniques in surgery, radiation and chemotherapy have been established (3). Identification of predictive markers is urgent to optimize treatment and reduce impairment for the individual patient. Incidence of oropharyngeal cancer, especially in the tonsil and tongue base, has steadily increased over the past two 
decades, notably in individuals 40-55 years of age. In contrast, incidence of HNSCC has decreased overall, which is attributed to a reduced prevalence of smoking (4-6). The most important risk factors are chronic tobacco and alcohol consumption. These appear to have a synergistic effect on the mucosal surfaces 'field cancerization'. Tobacco and alcohol use, poor oral hygiene and mutational loss of regulatory proteins remain important risk factors for head and neck tumors overall.

In the last 20 years there has been increasing awareness of a subset of squamous cell carcinomas induced by oncogenic forms of the human papilloma virus (HPV) of the squamous mucosa surface, which shows similarities to HPV-positive cervical SCC. High-risk HPV infection has been associated with anogenital carcinomas, including cervical, anal, vulvar and penile cancers, and more recently, breast cancer (7). In contrast to the falling incidence of head and neck cancers overall in the USA in recent years, the incidence of HPV-associated oropharyngeal cancer is rising, indicating the increased importance of the viral etiology (8-10). In the USA, $40-80 \%$ of oropharyngeal cancers are associated with HPV, whereas in Europe data are heterogenous and vary from $20 \%$ in countries with high tobacco and alcohol consumption to $90 \%$ in Sweden. This suggests that HPV is now the primary cause of tonsillar carcinoma in North America and Europe (11-14). Augmented incidence of HPV-associated oropharyngeal cancers represents an emerging viral epidemic of cancer. The International Agency for Research on Cancer (IARC) designates HPV as a risk factor for cancerogenesis of oropharyngeal cancer, and recent molecular and epidemiological data support the theory that high-risk types of HPV are responsible for a subset of oropahryngeal cancers (15-18). HPV-positive HNSCC may be responsible for oropharyngeal cancer and seems to be different from tobacco and alcoholinduced HNSCC (non-HPV) in its genetic, molecular and clinical profile. HPV-positive HNSCCs occur more often in younger patients with minimal tobacco exposure but greater exposure to marijuana, oral sex or multiple sexual partners (19-21), which is consistent with the known predominant means of HPV transmission via sexual contact $(21,22)$. HPV-positive HNSCC patients are often of higher socioeconomic status with better dentition, nutritional status and overall health compared to non-HPV HNSCC patients (23).

There is evidence that links this viral etiology to specific subtypes of HPV-associated HNSCC like HPV 16 and 18, especially to those arising from the tonsils and the tongue base.

HPV is a circular, double-stranded DNA virus. The viral genome, consisting of approximately 8,000 base pairs in size, encodes two regulatory proteins, three oncoproteins (E5, E6, and E7), and two structural capsid proteins (L1 and L2) (11). At present, the family of Papillomaviridae contains at least 200 genotypes or subtypes based on the ability to infect mucosal surfaces and based on genomic characteristics (13). These subtypes could be classified in low- and high-risk subpopulations based on their capacity to persist in basal mucosa cells, thereby avoiding the clearance of the immune system, and based on their potential for transformation by expression of viral oncogenes. Mucosal HPV infections are known to be associated with a spectrum of human diseases, ranging from benign papillomas (or warts) to invasive carcinomas such as cervical, vulvar, vaginal, anal, penile, recurrent benign papillomatous tumor of the upper airway, and more recently, HNSCC
(22-24). Clinical appearance is characterized by an exophytic strawberry-like lesion on gross inspection. HPV infection typically affects the basal layer of stratified squamous epithelium through structural breaks or microabrasion in the skin and the mucosa surface (25). Thus on one hand, the HPV infection evades the host immune system by residing in the basal epithelium, and on the other hand, viral oncogenes suppress pro-inflammatory signaling (26). The expression of the abovementioned oncogenes and the loss of function of several key regulatory proteins cause cell cycle progression, proliferation and dedifferentiation of the infected mucosa cells and thereby prove to be necessary for the induction and maintenance of the transformed phenotype $(27,28)$. These oncoproteins degrade and destabilize two major tumor suppressor proteins, p53 and $\mathrm{pRb}$, through ubiquitination and directly bind to multiple other host proteins. This further contributes to genetic instability by integration of its viral DNA into the host genome, leading to dysregulated expression of E6 and E7 $(28,29)$. In cervical cancer, the integration of viral DNA into the host genome is a crucial step for the progression from dysplasia to carcinoma. The biological pattern of HPV-positive oropharyngeal cancer is distinct from p53 degradation, retinoblastoma pathway inactivation, and p16 up-regulation. This is in contrast to oropharyngeal cancer, which is induced by repeated alcohol and tobacco exposure and is characterized by mutational loss of p16 and p53 genes, TP53 mutation and further down-regulation of CDKN2A (30-33), leading to uncontrolled cellular growth in up to $80 \%$ of HNSCC. The tumor suppressor gene p53 positively regulates the expression of angiogenic inhibitors and inhibits pro-angiogenic factors (34-36).

In addition to the etiological differences, HPV-positive cancers are different from HPV-negative cancers with regard to treatment response and survival outcome. HPV-positivity is considered to be a favorable prognostic biomarker for survival outcome. Data of retrospective analyses suggest that patients with HPV-positive oropharyngeal cancer have higher response rates to chemotherapy and radiation and therefore, have a significantly increased survival rate and locoregional control $(32,37,38)$. The literature offers different explanations for the overall advantage that patients with HPV-associated HNSCC have, such as augmented sensitivity to chemo- and radiotherapy and absence of exposure to tobacco and alcohol accompanied by field cancerization and presence of functional TP53 (37).

Important support functions, including promotion of tumor growth, angiogenesis and invasion, have been attributed to the different cell types populating the tumor stroma, like the endothelial cells, cancer-associated fibroblasts (CAFs), pericytes, and the infiltrating inflammatory cells that form the extracellular matrix (ECM). These cell types are functionally important for carcinogenesis because they provide proliferative and antiapoptotic regulatory factors, support tumor angiogenesis, and thus facilitate invasion (39-43). Various studies demonstrate the fundamental role of a concerted performance of genetically altered tumor cells interacting with ostensibly normal cell types in cancerogenesis. These factors constitute a concerted interaction of the tumor and its microenvironment $(44,45)$. A variety of angiogenic peptides released from tumor cells, macrophages and the extracellular matrix regulate the induction of tumor vascularization. Various functions, 
including endothelial cell migration, proliferation and capillary tubule formation, are executed by these factors.

As a potent endothelial mitogenic factor, the vascular endothelial growth factor (VEGF) activates the angiogenic switch in vivo and enhances vascular permeability. It is associated with an increase of tumor growth and angiogenesis in vivo in a mouse model (46-48). Cell proliferation, differentiation and migration of vascular cells is promoted by VEGF. Enhanced expression of VEGF has been detected in a number of malignant human tumors, including HNSCC $(49,50)$. VEGF expression in HNSCC tumors is strongly correlated with tumor angiogenesis and is inversely correlated with apoptosis $(51,52)$, which illustrates the anti-apoptotic potential of VEGF. For example, VEGF expression could be induced by loss or inactivation of tumor suppressor genes or overexpression of oncogenes. Several studies demonstrate that HPV16 oncoproteins E6 and E7 strongly stimulate various pro-angiogenic factors, including VEGF (53). Tumor angiogenesis, microvessel density and VEGF expression have been associated with poor prognosis of head and neck cancers (50-52). Inhibition of angiogenesis by blocking angiogenic cytokines or their pathways has become a major target not only in experimental cancer therapies.

Platelet-derived growth factor (PDGF) plays a crucial role in cellular interaction and phenotypic character of tissue architecture. The involvement of the different PDGF isoforms and the corresponding receptor plays a decisive role in regulating cell proliferation, chemotaxis and survival in normal cells as well as in the process of tumorigenesis by autocrine and paracrine stimulation. The biological activity of PDGF is linked to two tyrosine-kinase receptors: PDGF-R and PDGF-R $\beta$. The PDGF ligand binds to its receptor, thus providing intrinsic tyrosine-kinase activity. Hereby, receptor activation by dimerization and autophosphorylation is generated. The activation of the receptor induces signaling molecules that are responsible for the different biological effects of PDGF $(54,55)$. Previous studies demonstrated the increased expression of PDGF and the associated receptors in various malignant human tumors such as non-small cell lung (NSCLC) and prostate cancer $(56,57)$. PDGF promotes the establishment of a well-vascularized stroma and tumor proliferation by stimulating the process of angiogenesis (55-57) in fibrosarcomas (58), breast carcinoma (59), melanoma (60) and HNSCC (61).

The protein-tyrosine-kinase-receptors (PTKs) are essential elements of the intracellular signal transduction pathway that regulates cell growth, development and apoptosis. When mutations of various cancers occur, PTKs often disrupt the normal cell signaling pathway and may lead to malignant transformation (62). The superfamily of transmembrane receptor proteins is activated by binding to the corresponding ligand, such as the epidermal growth factor (EGF) or the transforming growth factor (TGF), and phosphorylation of the tyrosine-kinase domain. These second messenger molecules provide signals that enhance cell survival and increase cell proliferation (62). Unlike standard cytotoxic therapies that generally lack specificity for tumor cells and possess various toxic side effects, the targeted molecular approach exploits known molecular changes in neoplastic cells.

The tyrosine-kinase inhibitor imatinib (Gleevec $\left.{ }^{\circledR}\right)$, also known as STI571 (Novartis, Basel, Switzerland), is a small molecular agent belonging to the 2-phenylaminopyrimidine class. Initially designed to inhibit bcr-abl, c-kit and PGDFreceptor, it is currently established in the treatment of chronic myeloid leukemia (CML) (63-66).

The ability of imatinib to target several specific tyrosinekinases that are implicated in carcinogenesis and metastasis, like c-Abl and PDGF-R, makes it an ideal therapeutic agent with less toxic side effects. This therapeutic option is used currently in a limited number of cancers, such as gastrointestinal stromal tumors, melanomas, and various forms of lung cancers (66-70). Since these studies have demonstrated the proapoptotic effect of imatinib, this agent was subsequently evaluated in combination with different drugs, especially platinum-based chemotherapeutic drugs. Prior studies illustrated the potential synergistic effects of combining imatinib and carboplatin/cisplatin to regulate distinctively different biological targets and to elevate the therapeutic ratio in HNSCC $(71,72)$. Less is understood about the correlation of VEGF, PDGF and PDGF-R and positive HPV status of squamous cell carcinoma or the impact of tyrosine-kinase inhibitory therapy on angiogenic factors.

The purpose of this study was to evaluate the expression pattern of angiogenic factors (VEGF, PDGF and PDGF-R) in HPV-positive and negative squamous cell carcinoma and the vulnerability of anti-angiogenesis therapy dependent on the HPV status as a potential treatment modality compared to established platinum-based chemotherapeutic drugs. To our knowledge, this is the first report of a chemotherapeutic study to treat HNSCC and HPV 16-positive SCC with imatinib and carboplatin as single, active drugs and the first study to compare expression patterns of angiogenic peptides.

\section{Materials and methods}

Cell lines. The two different UM-SCC cell lines 11A and 14C were obtained from Dr T.E. Carey (University of Michigan, MI, USA). These cell lines originate from human HNSCC of the oropharynx and larynx. The CERV-196 cell line was established from a poorly differentiated squamous cell carcinoma of the cervix that is HPV 16 positive (CLS, Eppelheim, Germany). A CERV196 tumor is established in vitro from the xenotransplant cervix carcinoma MRI-H-196. The CERV196 cells were cultured in Eagle's minimum essential medium with $2 \mathrm{mM}$ L-glutamine and Earle's BSS adjusted to contain $1.5 \mathrm{~g} / 1$ sodium bicarbonate, $0.1 \mathrm{mM}$ non-essential amino acids, $1.0 \mathrm{mM}$ sodium pyruvate and $10 \%$ fetal bovine serum. Cell cultures were carried out at $37^{\circ} \mathrm{C}$ in a $5 \% \mathrm{CO}_{2}$ fully humidified atmosphere using Dulbecco's modified minimum essential medium (DMEM) (Fisher Scientific Co., Pittsburgh, PA, USA) supplemented with $10 \%$ fetal calf serum (FCS) and antibiotics (Life Technologies Inc., Gaithersburg, MD, USA). Imatinib was gratefully provided by the manufacturer (Novartis, Basel, Switzerland). Imatinib and carboplatin were stored at $4^{\circ} \mathrm{C}$ and dissolved in sterile water at the time of use. The HNSCC cell lines were incubated with different concentrations and combinations of imatinib $(18,30 \mu \mathrm{mol} / \mathrm{ml})$ and/or carboplatin (3, $7.5 \mu \mathrm{mol} / \mathrm{ml}$ ) for $2,3,5$, and up to 8-10 days. These different drug concentrations and combinations were selected after performing the alamarBlue (AbD Serotec, Oxford, UK) cell proliferation assay, quantitatively measuring proliferation of 
HNSCC tumor cell lines and establishing the relative cytotoxicity of the chemotherapeutic drugs examined. After the defined incubation time, the supernatants were collected in sterile tubes and stored at $-20^{\circ} \mathrm{C}$ until further analysis. After harvesting, cells were stored at $-80^{\circ} \mathrm{C}$ for PCR analysis.

Immunohistochemistry of PDGF- $\alpha / \beta$. Immunohistochemical studies were performed using a monoclonal rabbit anti-human antibody directed against PDGF- $\alpha / \beta$ (ACRIS Antibodies, Herford, Germany). The cells were cultured overnight on glass coverslips (Nunc, Wiesbaden, Germany) before immunohistochemistry was preformed. When $50 \%$ confluent, the cells were exposed to the different chemotherapeutic drug concentrations and different incubations periods (48-240 h) and underwent a fixation with acetone and alcohol (2:1). Afterwards, the cells were washed with phosphate-buffered saline (PBS). Adjacent cells were incubated with the peroxidase block (Dako Hamburg, Germany) for $30 \mathrm{~min}$. The cells were then washed three times with PBS for 5 min each time. This was followed by incubation with $10 \%$ sheep serum for $30 \mathrm{~min}$. Cells were exposed to the rabbit polyclonal antibody against PDGF- $\alpha / \beta$ as the primary antibody solution for $30 \mathrm{~min}$ at room temperature, using a working dilution of antibody to cells of 1:100. The incubated cell lines were refrigerated overnight. Afterwards, the cells were washed three times with PBS-buffer and incubated with a secondary antibody (antirabbit, Amersham, Freiburg, Germany) in a 1:100 solution for $45 \mathrm{~min}$ at room temperature. Following incubation, the cells were washed three times. Subsequently, cells were exposed to an enzyme suspension [AEC, aminoethylcarbazole (red) or DAB, diaminobenzidine (brown)] for 5-15 min. The sections received a counterstaining with Mayer's-hematoxylin for $30 \mathrm{sec}$. This procedure was followed by dehydration in graded ethanol and coverslipping.

The results of the observed immunohistochemical rates of PDGF expression were determined semiquantitatively. The staining intensity was described as follows: strong reactivity, $>80 \%$ of the cells were positive; moderate reactivity, $50-80 \%$ of the cells stained positive; weak reactivity, $<50 \%$ of the cells were positive; and negative immunostained cells ( $0 \%$ reactivity).

ELISA total PDGF-R- $\alpha / \beta$ and VEGF. After incubation in the 6-well chamber with the different chemotherapeutic drug concentrations, the cells were rinsed with PBS and then $350 \mu 1$ per well of lysis buffer were added. After gently agitating the lysed cells with a vortex at $2-8^{\circ} \mathrm{C}$ for $30 \mathrm{~min}$ and microcentrifuging at $14,000 \mathrm{xg}$ for $5 \mathrm{~min}$, the supernatant was transferred into a clean tube.

VEGF concentrations were determined by the enzymelinked immunosorbent assay (ELISA) technique (R\&D Systems, Wiesbaden, Germany). The system used a solid-phase monoclonal antibody and an enzyme-linked polyclonal antibody raised against recombinant VEGF165. The specificity of antihuman VEGF antibodies used in the ELISA kit was examined by sodium dodecyl sulfate polyacrylamide gel electrophoresis (SDS-PAGE) followed by Western blotting. According to the manufacturer's directions, each ELISA m was performed in $100 \mu \mathrm{l}$ of supernatant. All analyses and calibrations were carried out in duplicate. The calibrations on each microtiter plate included recombinant human VEGF standards that were provided in the kit. Optical density was determined using a microplate reader at a wavelength of $450 \mathrm{~nm}$. Wavelength correction was set to $540 \mathrm{~nm}$ and concentrations were reported as $\mathrm{pg} / \mathrm{ml}$. The interassay coefficient of variation reported by the manufacturer varied from 6.2 to $8.8 \%$ when VEGF concentrations ranged between 50 and $1,000 \mathrm{pg} / \mathrm{ml}$.

The DuoSet IC ELISA (R\&D Systems) measures human PDGF-R $\alpha / \beta$ by development of a sandwich ELISA with an immobilized capture antibody specific for human PDGF-R, which binds both tyrosine-phosphorylated and unphosphorylated PDGF-R. After washing away the unbound material, a biotinylated detection antibody specific for total human PDGF-R is used to detect tyrosine-phosphorylated and unphosphorylated PDGF-R, utilizing a standard strepavidinHRP format.

The capture antibody was diluted to the concentration 1:180 $(4 \mu \mathrm{g} / \mathrm{ml})$. The diluted capture antibody was carried out in $100 \mu \mathrm{l}$ per well and the plate was sealed and incubated overnight. Afterwards, the contents of each well were aspirated and the wells were washed three times with $400 \mu \mathrm{l}$ Tween wash buffer. The plates were blocked by adding $300 \mu$ l block buffer to each well and incubating at room temperature for 1-2 $\mathrm{h}$. According to the manufacturer's directions, each ELISA measured $100 \mu \mathrm{l}$ of supernatant of the sample. This was followed by washing with Tween buffer as described. After diluting the detection antibody to a concentration of $500 \mathrm{ng} / \mathrm{ml}$, the detection antibody $(100 \mu \mathrm{l})$ was added to each well with an incubation period of $2 \mathrm{~h}$. Again cells were washed. Adding $100 \mu \mathrm{g}$ of streptavidinHRP to each well was followed by incubation for $20 \mathrm{~min}$ at room temperature. Afterwards, $100 \mu \mathrm{l}$ of substrate solution was added to each well for 20 min followed by $50 \mu 1$ of stop solution. Optical density was determined using a microplate reader at a wavelength of $450 \mathrm{~nm}$. Wavelength correction was set to $540 \mathrm{~nm}$ and concentrations were reported as $\mathrm{pg} / \mathrm{ml}$. All analyses and calibrations were carried out three times.

Statistical analysis. Statistical analysis was performed in cooperation with Dr Chr. Weiss, Institute of Biomathematics, Faculty of Medicine, Mannheim. All data were subjected to the means procedure. A p-value $\leq 0.05$ was considered statistically significant. The statistical tests performed were the two-coefficient variance analysis (SAS Statistics, Cary, NC) and the Dunnett's test.

\section{Results}

Immunohistochemistry for PDGF- $\alpha$ in HNSCC 11A, $14 C$ and CERV196. The immunohistochemically-detected protein expression of PDGF- $\alpha$ was assessed by two independent observers in order to semiquantitatively calculate the rates of protein expression. All tumor cell lines, regardless of HPV status, expressed PDGF- $\alpha$ to a similar extent. The controls showed high reactivity against PDGF- $\alpha$. Furthermore, in HNSCC $11 \mathrm{~A}$ and $14 \mathrm{C}$ cells a decreased reactivity of PDGF expression was detected, dependent on an extended incubation period with imatinib. CERV196 cells, an HPV 16 positive carcinoma cell line, showed a lower reduction of PDGF- $\alpha$ expression under increased imatinib concentrations and thus was considered to be less sensitive to anti-angiogenic therapy. 
Table I. Grading of immunostaining for PDGF- $\alpha$.

\begin{tabular}{|c|c|c|c|c|}
\hline & \multicolumn{4}{|c|}{ Immunostaining index } \\
\hline & $48 \mathrm{~h}$ & $72 \mathrm{~h}$ & $120 \mathrm{~h}$ & $240 \mathrm{~h}$ \\
\hline \multicolumn{5}{|l|}{ Control group } \\
\hline HNSCC 14C & $++(3 / 3)$ & $+++(2 / 3)$ & $++(2 / 3)$ & $+++(3 / 3)$ \\
\hline HNSCC 11A & $++(3 / 3)$ & $++(2 / 3)$ & $+++(3 / 3)$ & $+++(2 / 3)$ \\
\hline CERV196 & $++(3 / 3)$ & $++(3 / 3)$ & $+++(3 / 3)$ & $++(2 / 3)$ \\
\hline \multicolumn{5}{|c|}{ Carboplatin $3 \mu \mathrm{mol}$} \\
\hline HNSCC 14C & $+(1 / 3)$ & $++(2 / 3)$ & $++(2 / 3)$ & $++(1 / 3)$ \\
\hline HNSCC 11A & $++(2 / 3)$ & $++(1 / 3)$ & $++(1 / 3)$ & $+++(2 / 3)$ \\
\hline CERV196 & $+++(2 / 3)$ & $+++(1 / 3)$ & $+++(3 / 3)$ & $+++(2 / 3)$ \\
\hline \multicolumn{5}{|c|}{ Carboplatin $7.5 \mu \mathrm{mol}$} \\
\hline HNSCC 14C & $++(2 / 3)$ & $++(2 / 3)$ & $++(2 / 3)$ & $++(2 / 3)$ \\
\hline HNSCC 11A & $++(2 / 3)$ & $+++(2 / 3)$ & $++(1 / 3)$ & $+++(1 / 3)$ \\
\hline CERV196 & $+++(3 / 3)$ & $+++(3 / 3)$ & $+++(2 / 3)$ & $+++(2 / 3)$ \\
\hline \multicolumn{5}{|c|}{ Imatinib $18 \mu \mathrm{mol}$} \\
\hline HNSCC 14C & $++(2 / 3)$ & $+(2 / 3)$ & $+(1 / 3)$ & $+(1 / 3)$ \\
\hline HNSCC 11A & $++(1 / 3)$ & $++(1 / 3)$ & $++(1 / 3)$ & $++(2 / 3)$ \\
\hline CERV196 & $+++(3 / 3)$ & & $++(1 / 3)$ & $++(2 / 3)$ \\
\hline \multicolumn{5}{|c|}{ Imatinib $30 \mu \mathrm{mol}$} \\
\hline HNSCC 14C & $++(2 / 3)$ & $0(1 / 3)$ & $0(1 / 3)$ & $0(1 / 3)$ \\
\hline HNSCC 11A & $+(2 / 3)$ & $+(2 / 3)$ & $+(2 / 3)$ & $+(1 / 3)$ \\
\hline CERV196 & $++(2 / 3)$ & $++(1 / 3)$ & $++(2 / 3)$ & $+(1 / 3)$ \\
\hline
\end{tabular}

0 , No positive cells; + , weak immunostaining; ++, moderate immunostaining; +++ , strong immunostaining. (x/3), number of positive cell lines out of the HNSCC lines 14C, 11A and CERV196.

Immunostaining was localized in the cytoplasm of the cells (Fig. 1, Table I).

ELISA for VEGF expression on HNSCC 14C, 11A and CERV196. VEGF levels showed a consistent trend towards significantly decreased expression that was dependent on the agent added for an extended incubation period, whereas increased concentrations of the added agents had no significant influence. For the purpose of simplification, only the $30 \mu \mathrm{mol} / \mathrm{ml}$ concentration of imatinib and the $7.5 \mu \mathrm{mol} / \mathrm{ml}$ concentration of carboplatin are presented and discussed in the results. Furthermore, the application of carboplatin had no influence on VEGF expression levels. In addition to performing descriptive imaging of the results (mean values and percent reduction of the effect variable compared to control), a two-coefficient analysis and the Dunnett's test were conducted.

For HNSCC 11A cells, there was no significant reduction of VEGF and no evident dose- or incubation period-dependent influence of carboplatin when compared to the negative control. Effects of carboplatin as a single agent on VEGF expression of HNSCC 14C were negligible; only a single statistical outlier after 5 and 8 days with $p<0.04$ and $p=0.01$ was detected. Again, no dose- or incubation period-dependent effect was statistically detectable. The influence of carboplatin on VEGF expression was also insignificant for the CERV16 cell line (Table II, Fig. 2).

In contrast to the carboplatin application, non-HPV associated HNSCC tumor cell lines (HNSCC 11A and 14C) incubated with imatinib showed a significant reduction of VEGF expression levels in an incubation period-dependent correlation (especially for HNSCC 11A) when compared to the negative control. The degree of concentration had no significant influence on expression of VEGF.

Within the timeframe of 48-192 h, there was an insignificant reduction of VEGF expression levels in CERV196. The CERV 196 tumor cells seemed to be less vulnerable towards PTK inhibitory therapy according to suppression of the expression of VEGF. Only after $240 \mathrm{~h}$ of incubation did the CERV196 cells show a decreased VEGF expression with significant correlation to negative controls $(\mathrm{p}<0.0001)$. Compared to non-HPV associated tumor cell lines after the same incubation time (240 h), CERV196 proved to have the highest decrease of VEGF ( $\mathrm{p}<0.0001)$ (Table II).

The statistical comparison of the efficacy of the different chemotherapeutic applications clarifies the significant advantage of imatinib in suppressing VEGF levels in HNSCC 11A and 14C. In CERV196 cells, imatinib prevailed against carboplatin after an incubation period of more than $120 \mathrm{~h}$ (Table II). 

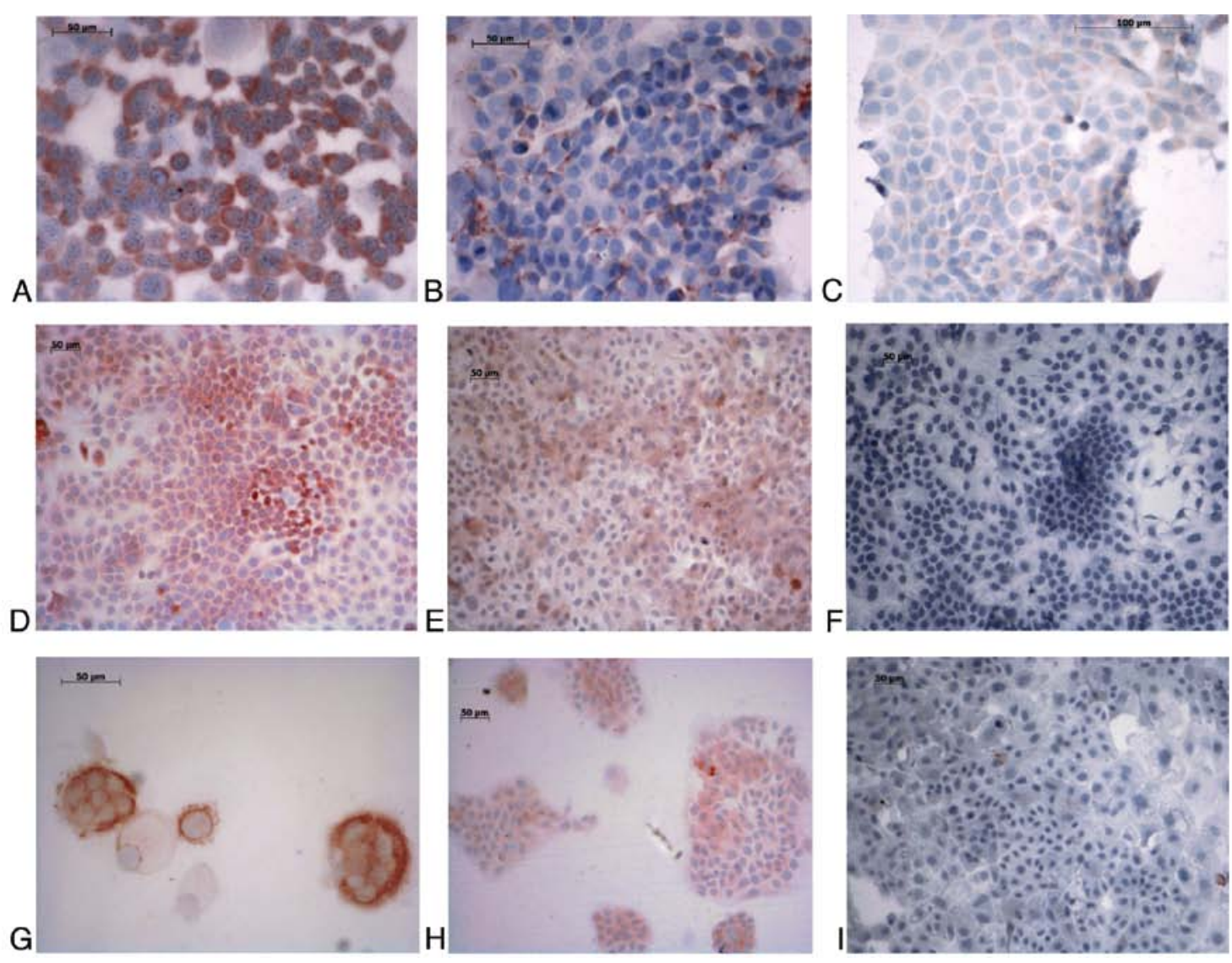

Figure 1. (A) Negative control of HNSCC $11 \mathrm{~A}$ cells and positive immunohistochemical reactivity against PDGF- $\alpha$ after incubation with imatinib (30 $\mu \mathrm{mol} / \mathrm{ml})$ for (B) 120 and (C) $240 \mathrm{~h}$. (D) Negative control of HNSCC 14B cells and positive immunohistochemical reactivity against PDGF- $\alpha$ after incubation with imatinib (30 $\mu \mathrm{mol} / \mathrm{ml}$ ) for (E) 120 and (F) $240 \mathrm{~h}$. (G) Negative control of CERV196 with strong immunoreactivity with typical growth pattern in tumor cell colonies. (H and I) Decreasing immunoreactivity was detected with a prolonged incubation period (120 and $240 \mathrm{~h})$ with imatinib $(30 \mu \mathrm{mol} / \mathrm{ml})$.

Table II. VEGF expression in ELISA.

\begin{tabular}{|c|c|c|c|c|c|}
\hline \multirow[b]{2}{*}{ Time of incubation (h) } & \multirow[b]{2}{*}{ Control group } & \multicolumn{2}{|c|}{ Carboplatin $7.5 \mu \mathrm{mol} / \mathrm{ml}$} & \multicolumn{2}{|c|}{ Imatinib $30 \mu \mathrm{mol} / \mathrm{ml}$} \\
\hline & & Mean value & p-value & Mean value & $\mathrm{p}$-value \\
\hline \multicolumn{6}{|l|}{ HNSCC 11A } \\
\hline 48 & 2044.5 & 2036 & 0.9 & 1871 & $<0.0001$ \\
\hline 72 & 2003.6 & 1993.3 & 0.9 & 1580 & $<0.0001$ \\
\hline 120 & 2030 & 1942.6 & 0.3 & 1536 & $<0.0001$ \\
\hline 240 & 2014.5 & 1934.3 & 0.2 & 1399.6 & $<0.00001$ \\
\hline \multicolumn{6}{|l|}{ HNSCC $14 \mathrm{C}$} \\
\hline 48 & 1969 & 1850.3 & 0.9 & 1939.6 & 0.9 \\
\hline 72 & 2111 & 2063.6 & 0.5 & 1656.3 & $<0.0001$ \\
\hline 120 & 2108 & 1823.6 & $<0.04$ & 1729.3 & $<0.0001$ \\
\hline 240 & 2224.5 & 1954 & 0.9 & 1673 & 0.0001 \\
\hline \multicolumn{6}{|l|}{ CERV196 } \\
\hline 48 & 2060.6 & 1940 & 0.8 & 1920.3 & 0.47 \\
\hline 72 & 2064.6 & 2029.6 & 0.9 & 1845.3 & 0.26 \\
\hline 120 & 1823.6 & 1847.6 & 0.6 & 1533 & 0.2 \\
\hline 240 & 2048.3 & 1877.6 & 0.09 & 726.6 & $<0.0001$ \\
\hline
\end{tabular}

VEGF expression in HNSCC 11A, 14C and CERV196 after different periods of incubation. Mean values and statistical correlation compared to the negative control ( $\mathrm{p}$-value, Dunnett's test). Bold indicates statistically significant differences. 
VEGF-expression in HNSCC 11A

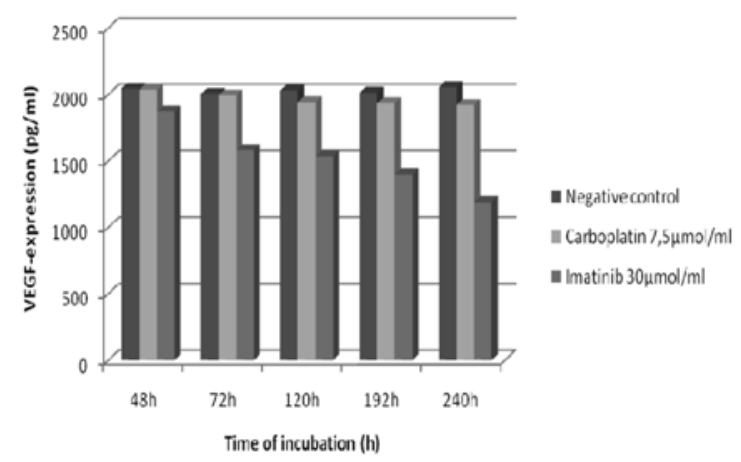

VEGF-expression in HNSCC $14 C$

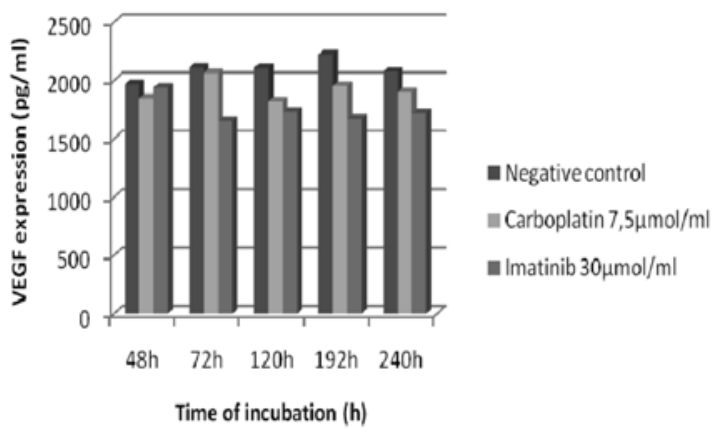

VEGF-expression in CERV196

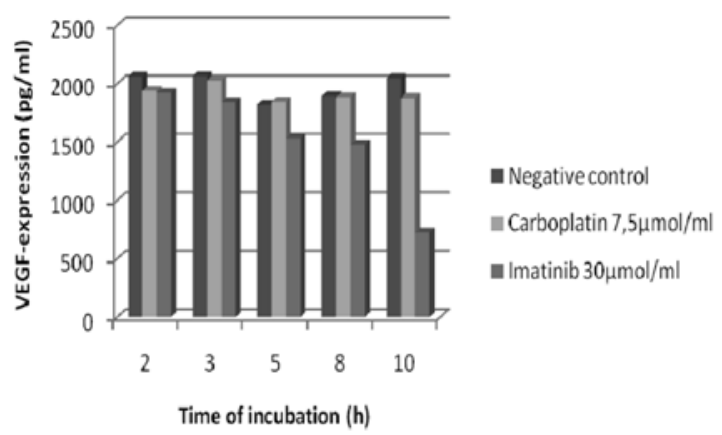

Figure 2. VEGF expression in HNSCC 11A, 14C and CERV196 cells. Decreased VEGF expression after a prolonged incubation period $(48,72$, $120,192$ and $240 \mathrm{~h})$.

ELISA of PDGF-R $\alpha / \beta$ expression in HNSCC 14C, 11A and CERV196. Carboplatin had no statistically significant impact on the expression of PDGF-R $\alpha / \beta$, regardless of the dosage or incubation time. Only a moderate suppression of PDGF-R $\beta$ for CERV196 was notable (Tables III and IV, Figs. 3 and 4).

In contrast, imatinib as a tyrosine-kinase inhibitor showed a statistically significant reduction of PDGF-R $\alpha / \beta$ levels in all incubated tumor cell lines, irrespective of HPV status, depending on the length of incubation (Tables III and IV). Several times a decent reduction of the initial value of the PDGF-R expression level was illustrated. There was a slight but not statistically significant difference in suppression of the PDGF-R expression when comparing HPV-positive with HPV-negative tumor cell lines (Figs. 3 and 4).

\section{Discussion}

Despite the general decrease in incidence of HNSCC that can be attributed to a reduced prevalence of chronic alcohol and tobacco exposure, the incidence of squamous cell carcinoma of the oropharynx and oral cavity is rising. A growing number of malignant lesions of the tonsils and tongue base is particularly notable (4-6,11-14). Apart from the above-mentioned risk factors, a growing body of evidence highlights an infectious etiology initiating the cancerogenesis of HNSCC. Epidemiological data suggest that an infection of oncogenic high-risk papilloma virus in carcinogenesis of HNSCC is responsible for a subset of oropharyngeal cancer. HNSCC is a heterogeneous group of epithelial malignancies that differ in their aggressiveness, treatment response and clinical outcome. Despite the established therapeutic options over the last decades, HNSCC tumors still have poor prognosis. New treatment modalities are urgently necessary to improve the clinical outcome, long-term survival and quality of life for head and neck tumor patients. HPV-positive SCC appears to be different from HPV-negative HNSCC in molecular, epidemiological and clinical aspects as well as in the overall survival rate due to increased chemotherapeutic sensitivity as described in the literature $(32,37)$. Less is known about HPV-positive HNSCC tumor biology, carcinogenesis and the role of the immune system and cellular pattern in new treatment regimes. One strategy in novel approaches to cancer therapy is the inhibition of angiogenesis of malignant tumors. Various studies detect the induced angiogenic response in vivo, and the correlation between high microvessel counts and recurrent or metastatic disease in HNSCC $(51,52)$. A variety of angiogenic factors released from tumor cells and the extracellular matrix regulate the induction of tumor vascularization.

PDGF promotes the establishment of a well-vacularized stroma and thus tumor proliferation by stimulating the angiogenesis process (55-57) in melanoma (58), fibrosarcoma (59), breast carcinoma (60) and squamous carcinoma (61). VEGF, as another fundamental stimulator of angiogenesis, enhances vascular permeability (46) and is associated with an increase of tumor growth and angiogenesis. Disrupted PDGF and PDGF-R signaling pathways could also prove to be a functional element in HNSCC tumor biology and correlate inversely with the clinical outcome in patients who suffer from $\operatorname{HNSCC}(48,49)$. Thus, these angiogenic peptides might function as surrogate markers in cases of dysregulated expression with poor overall survival and therefore, could be potential clinical targets in molecular-specific therapy. Previous studies have demonstrated the statistically significant antiproliferative impact of imatinib in conjunction with platinum-based chemotherapy in HNSCC in vitro by reducing VEGF and PDGF and the corresponding tyrosine-kinase receptor $(71,72)$. The precise mechanism of synergism remains unclear. It is postulated that the synergism is the result of an alteration of PTK expression, modulation of the phosphorylation status of the PTK receptor, or change in the apoptotic threshold by promoting pro-apoptotic balance of Bcl-2 family regulators.

Strong expression levels of VEGF could be detected in the HPV-positive SCC tumor cell line (CERV196) through immunohistochemistry and ELISA methods. Compared to HPV-positive SCC, HPV-negative HNSCC tumor cells were 
Table III. PDGF-R $\alpha$ expression in ELISA.

\begin{tabular}{|c|c|c|c|c|c|}
\hline \multirow[b]{2}{*}{ Time of incubation (h) } & \multirow[b]{2}{*}{ Control group } & \multicolumn{2}{|c|}{ Carboplatin $7.5 \mu \mathrm{mol} / \mathrm{ml}$} & \multicolumn{2}{|c|}{ Imatinib $30 \mu \mathrm{mol} / \mathrm{ml}$} \\
\hline & & Mean value & $\mathrm{p}$-value & Mean value & $\mathrm{p}$-value \\
\hline \multicolumn{6}{|l|}{ HNSCC 11A } \\
\hline 48 & 895.6 & 792.8 & 0.5 & 555.6 & 0.02 \\
\hline 72 & 878.2 & 929 & 0.9 & 775 & 0.5 \\
\hline 120 & 825.3 & 826.3 & 1 & 706.6 & 0.9 \\
\hline 192 & 941.3 & 919.3 & 0.9 & 795.6 & 0.4 \\
\hline 240 & 914.3 & 1000 & 0.9 & 589 & 0.045 \\
\hline \multicolumn{6}{|l|}{ HNSCC $14 \mathrm{C}$} \\
\hline 48 & 842.1 & 858 & 0.4 & 911.3 & 0.8 \\
\hline 72 & 981 & 845.6 & 0.6 & 744.3 & 0.4 \\
\hline 120 & 1150.6 & 1034 & 0.1 & 699.6 & 0.09 \\
\hline 192 & 1028 & 987.1 & 0.9 & 576 & 0.01 \\
\hline 240 & 1000 & 925 & 0.3 & 557 & 0.001 \\
\hline \multicolumn{6}{|l|}{ CERV196 } \\
\hline 48 & 1477.95 & 1494.5 & 0.6 & 1431 & 0.9 \\
\hline 72 & 1602.4 & 1680 & 0.8 & 1457 & 0.4 \\
\hline 120 & 1779.0 & 1754.1 & 1 & 1556.4 & 0.09 \\
\hline 192 & 1756 & 1596.6 & 0.2 & 1462.8 & 0.01 \\
\hline 240 & 1852.3 & 1657.4 & 0.3 & 1422.1 & 0.001 \\
\hline
\end{tabular}

PDGF-R $\alpha$ expression in HNSCC 11A, 14C and CERV196 after different periods of incubation. Mean values and statistical correlation compared to the negative control ( $\mathrm{p}$-value, Dunnett's test). Bold indicates statistically significant differences.

Table IV. PDGF-R $\beta$ expression in ELISA.

\begin{tabular}{|c|c|c|c|c|c|}
\hline \multirow[b]{2}{*}{ Time of incubation (h) } & \multirow[b]{2}{*}{ Control group } & \multicolumn{2}{|c|}{ Carboplatin $7.5 \mu \mathrm{mol} / \mathrm{ml}$} & \multicolumn{2}{|c|}{ Imatinib $30 \mu \mathrm{mol} / \mathrm{ml}$} \\
\hline & & Mean value & p-value & Mean value & p-value \\
\hline \multicolumn{6}{|l|}{ HNSCC 11A } \\
\hline 48 & 80.7 & 80.4 & 0.9 & 60.5 & 0.2 \\
\hline 72 & 85.9 & 75.4 & 0.6 & 65.1 & 0.1 \\
\hline 120 & 101.7 & 76.4 & 0.3 & 47.9 & 0.01 \\
\hline 192 & 78.4 & 73 & 0.5 & 54.6 & 0.05 \\
\hline 240 & 101.6 & 74.3 & 0.07 & 47.1 & 0.001 \\
\hline \multicolumn{6}{|l|}{ HNSCC 14C } \\
\hline 48 & 51.2 & 40.7 & 0.1 & 31.4 & 0.001 \\
\hline 72 & 50.5 & 39.3 & 0.07 & 35.6 & 0.004 \\
\hline 120 & 70.7 & 61.1 & 0.2 & 32.8 & $<0.0001$ \\
\hline 192 & 96.8 & 69.4 & 0.0005 & 30.4 & $<0.0001$ \\
\hline 240 & 75.9 & 44.7 & 0.6 & 33.6 & 0.005 \\
\hline \multicolumn{6}{|l|}{ CERV196 } \\
\hline 48 & 71.6 & 94.5 & 0.02 & 96.4 & 0.09 \\
\hline 72 & 102.8 & 105.4 & 0.7 & 83.1 & 0.1 \\
\hline 120 & 103.5 & 78.1 & 0.09 & 59.1 & 0.006 \\
\hline 192 & 101.1 & 82.3 & 0.1 & 55.8 & 0.004 \\
\hline 240 & 126.4 & 85.6 & 0.01 & 47.4 & 0.0002 \\
\hline
\end{tabular}

PDGF-R $\beta$ expression in HNSCC 11A, 14C and CERV196 after different periods of incubation. Mean values and statistical correlation compared to the negative control ( $\mathrm{p}$-value, Dunnett's test). Bold indicates statistically significant differences. 
PDGF-R $\alpha$ expression in HNSCC 11A

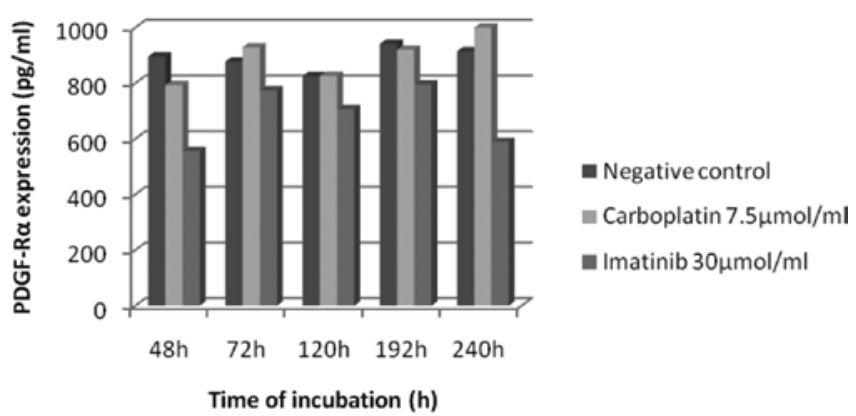

PDGF-R $\alpha$ expression in HNSCC 14C

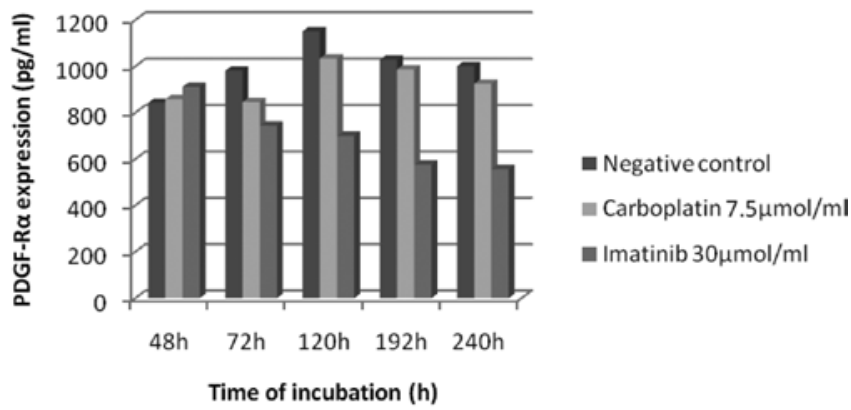

PDGF-R $\alpha$ expression in CERV196

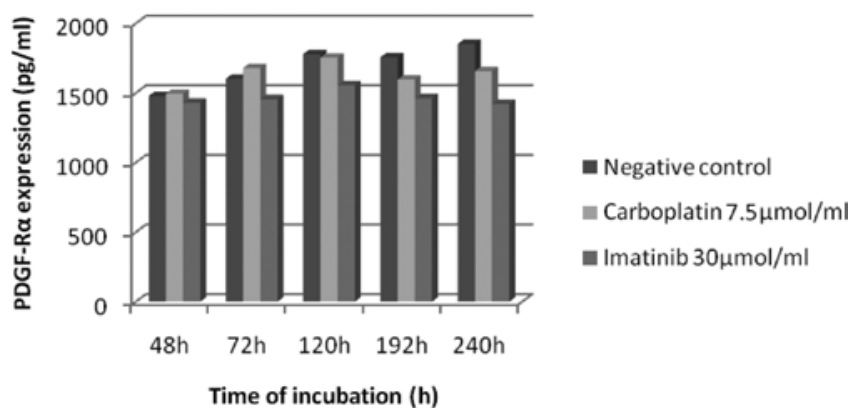

Figure 3.PDGF-R $\alpha$ expression in HNSCC 11A, 14C and CERV196 cells. Decreased PDGF-R $\alpha$ expression after a prolonged incubation period $(48,72$, 120,192 and $240 \mathrm{~h}$ ).

more susceptible to incubation time-related suppression of VEGF with imatinib treatment $(\mathrm{p}<0.0001)$. HPV 16 positive squamous cell carcinoma seemed to be less vulnerable to imatinib-associated VEGF suppression. A predominance of imatinib-related VEGF suppression in CERV196 tumor cells could only be proven after a prolonged incubation time. Dose escalation of the applicated drugs had no statistically significant influence on VEGF expression in either the CERV196 or the HNSCC tumor cell line. As previously documented, carboplatin had no impact on expression patterns in squamous cell carcinoma (84). VEGF expression in HNSCC tumors strongly correlated with tumor angiogenesis and inversely correlated with apoptosis $(53,54)$, which illustrates the antiapoptotic potential of VEGF. By significantly suppressing VEGF expression in HPV positive/negative SCC, imatinib as
PDGF-R $\beta$ expression in HNSCC 11A

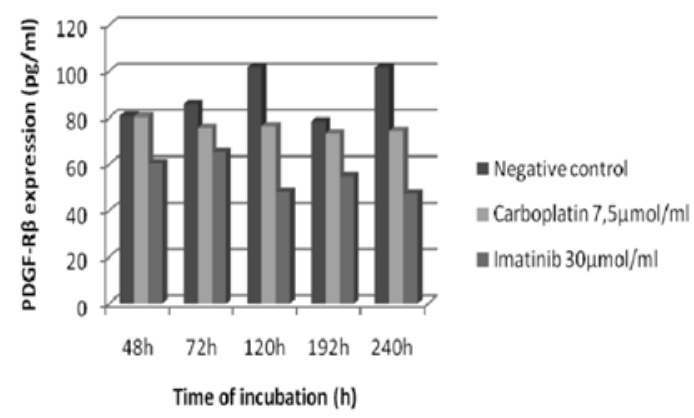

PDGF-Rß expression in HNSCC $14 C$

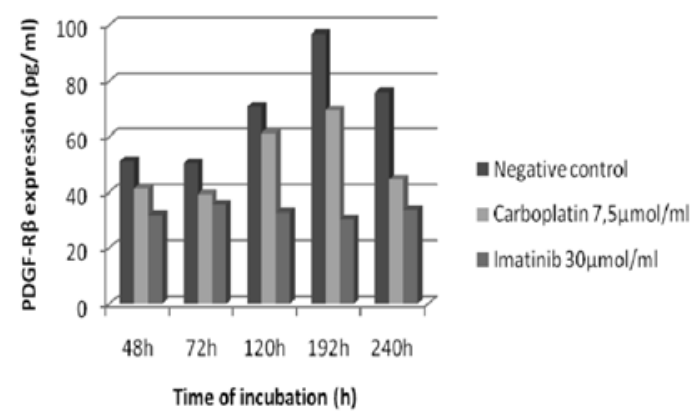

PDGF-R $\beta$ expression in CERV196

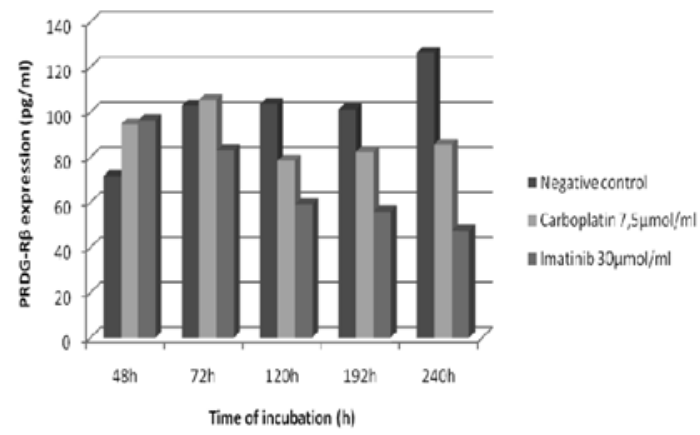

Figure 4. PDGF-R $\beta$ expression in HNSCC 11A, 14C and CERV196 cells. Decreased PDGF-R $\beta$ expression after a prolonged incubation period $(48,72$, 120,192 and $240 \mathrm{~h}$ ).

a molecular-specific agent might improve the clinical outcome of patients suffering from a malignant tumor disease in the future.

Furthermore, we detected a significant reduction of PDGF-R $\alpha / \beta$ expression in an incubation time-dependant manner for imatinib in all incubated tumor cell lines, irrespective of HPV status, whereas the application of a platinum-based chemotherapy had no influence on the expression of the tyrosine-kinase receptor. PDGF and the corresponding receptor PDGF-R $\alpha / \beta$ are fundamental effectors in tumor cell growth, angiogenesis and survival of the tumor $(64,65,77,78)$, and therefore, are warrantors of the carcinogenesis progression. These data suggest that a reduction of PDGF and PDGF-R expression levels by imatinib induces a reciprocal suppression of VEGF concentration within a blocked paracrine stimulation 
(73). One hypothesis for the mutual interaction of these angiogenic peptides is the PDGF/PDGF-R autocrine secretion of the tumor and a stimulated paracrine VEGF loop (73). The interference of angiogenic effectors could be specifically targeted by imatinib. Matei et al (73) and Vlahovic et al (70) also detected this correlation of the potential paracrine VEGF expression induction with the increased PDGF/PDGF-R activation in ovarian cancer and in a xenograft small lung cell cancer model. The suggested underlying mechanism for the imatinib associated-VEGF suppression is the sole inhibition of the interaction of PDGF-ligand and its corresponding tyrosine-kinase receptor $(72,73)$.

In summary, this study demonstrates the efficacy of imatinib as a single agent in the significant suppression of VEGF expression in HPV-negative HNSCC in an incubation time-related context. Viral oncogene transformed squamous cell carcinoma (CERV196) cells were characterized by reduced susceptibility for imatinib-altered VEGF expression. The observed effect of decreased VEGF expression in HPV-negative HNSCC could only be verified for HPV-positive SCC after a prolonged incubation time. According to PDGF$\mathrm{R} \alpha / \beta$ expression patterns, a statistically significantly reduction was notable for HPV transformed squamous cell carcinoma as already published for HPV- negative HNSCC (84).

This marked effect of selective tyrosine-kinase inhibitory therapy in suppressing VEGF, PDGF and PDGF-R as warrantors of tumor vascularization and therefore promoters of carcinogenesis in HPV-positive tumor cell lines justifies an eventual extension of this study to an animal model. Further studies are planned to investigate this observation in HPV-positive HNSCC in vitro. The implementation of a selective molecular anti-angiogenic therapy in established chemotherapeutic regimens might enhance the efficacy of platinum-based chemotherapy without an increased toxicity profile and could thereby improve clinical outcome in HNSCC, irrespective of HPV status.

\section{Acknowledgements}

The authors would like to thank Petra Prohaska for her excellent technical assistance, Dr C. Weiss for her assistance in statistical analysis, and Novartis Pharma (Basel, Switzerland) for kindly providing imatinib.

\section{Refetrences}

1. Parkin DM, Bray F, Ferlay J and Pisani P: Global cancer statistics, 2002. CA Cancer J Clin 55: 74-108, 2002.

2. IARC(International Agency for Research on Cancer): GLOBOCAN database. Available at www-dep.iarc.fr, 2002.

3. Ries LAG, Eisner MP, Kosary CL, et al: SEER Cancer Statistics Review: 1975-2002. National Cancer Institute, Bethesda, MD, http://seer.cancer.gov/csr/1975_2002/, based on November 2004 SEER data submission, posted to the SEER web site in 2005.

4. Pindborg J, Zheng KH, Kong CR, et al: Pilot survey of oral mucosa in areca (betel) nut chewers on Hainan Island of the People's Republic of China. Community Dent Oral Epidemiol 12: 195-196, 1984.

5. Licitra L, Bernier J, Grandi C, Merlano M, Bruzzi P and Lefebvre JL: Cancer of the oropharynx. Crit Rev Oncol Hematol 41: 107-122, 2002.

6. Sturgis EM and Cinciripini PM: Trends in head and neck cancer incidence in relation to smoking prevalence: an emerging epidemic of human papillomavirus-associated cancers? Cancer 110: 1429-1435, 2007.
7. Romanitan M, Näsman A, Ramqvist T, et al: Human papillomavirus frequency in oral and oropharyngeal cancer in Greece. Anticancer Res 28: 2077-2080, 2008.

8. Chaturvedi AK, Engels EA, Anderson WF and Gillison ML: Incidence trends for human papillomavirus-related and -unrelated oral squamous cell carcinomas in the United States. J Clin Oncol 26: 612-619, 2008.

9. Gillison ML, D'Souza G, Westra W, et al: Distinct risk factor profiles for human papillomavirus type 16-positive and human papillomavirus type 16-negative head and neck cancers. J Natl Cancer Inst 100: 407-420, 2008.

10. Shiboski CH, Schmidt BL and Jordan RC: Tongue and tonsil carcinoma: increasing trends in the US population ages 20-44 years. Cancer 103: 1843-1849, 2005.

11. Näsman A, Attner P, Hammarstedt L, et al: Incidence of human papilloma virus (HPV) positive tonsillar carcinoma in Stockholm, Sweden: an epidemic of viral induced carcinoma? Int J Cancer 125: 362-366, 2009.

12. Dilianis T, Näsman A, Attner P, et al: Human papillomavirus in tonsillar cancer, an epidemic of viral carcinoma. 25th international Papillomavirus Conferenc, Malmö, Sweden, May 8-14, O17.04. 2009.

13. Hansson BG, Rosenquist K, Antonsson A, et al: Strong association between infection with human papillomavirus and oral and oropharyngeal squamous cell carcinoma: a population-based case control study in southern Sweden. Acta Otolaryngol 125: 1337-1344, 2005

14. Brandsma JL and Abramson AL: Association of papillomavirus with cancers of the head and neck. Arch Otolaryngol Head Neck Surg 115: 621-625, 1989.

15. Kreimer AR, Clifford GM, Boyle P and Franceschi S: Human papillomavirus types in head and neck squamous cell carcinomas worldwide: a systematic review. Cancer Epidemiol Biomarkers Prev 14: 467-475, 2005.

16. Applebaum KM, Furniss CS, Zeka A, et al: Lack of association of alcohol and tobacco with HPV16-associated head and neck cancer. J Natl Cancer Inst 99: 1801-1810, 2007.

17. Pintos J, Black MJ, Sadeghi N, et al: Human papillomavirus infection and oral cancer: a case-control study in Montreal, Canada. Oral Oncol 44: 242-250, 2008.

18. Smith EM, Ritchie JM, Summersgill KF, et al: Human papillomavirus in oral exfoliated cells and risk of head and neck cancer. J Natl Cancer Inst 96: 449-455, 2004.

19. Koch WM, Lango M, Sewell D, Zahurak M and Sidransky D: Head and neck cancer in non-smokers: a distinct clinical and molecular entity. Laryngoscope 109: 1544-1551, 1999.

20. Tachezy R, Klozar J, Rubenstein L, et al: Demographic and risk factors in patients with head and neck tumors. J Med Virol 81: $878-887,2009$

21. Termine N, Panzarella V, Falaschini S, et al: HPV in oral squamous cell carcinoma vs head and neck squamous cell carcinoma biopsies: a meta-analysis (1988-2007). Ann Oncol 19: 1681-1690, 2008.

22. Smith EM, Hoffman HT, Summersgill KS, Kirchner HL, Turek LP and Haugen TH: Human papillomavirus and risk of oral cancer. Laryngoscope 108: 1098-1103, 1998.

23. Gillison ML, Koch WM, Capone RB, et al: Evidence for a causal association between human papillomavirus and a subset of head and neck cancers. J Natl Cancer Inst 92: 709-720, 2000.

24. D'Souza G, Kreimer AR, Viscidi R, et al: Case control study of human papillomavirus and oropharyngeal cancer. N Engl J Med 356: 1944-1956, 2007.

25. Pyeon D, Lambert PF and Ahlquist P: Production of infectious human papillomavirus independently of viral replication and epithelial cell differentiation. Proc Natl Acad Sci USA 102: 9311-9316, 2005.

26. Wiest T, Schwarz E, Enders C, Flechtenmacher C and Bosch FX: Involvement of intact HPV16 E6/E7 gene expression in head and neck cancers with unaltered p53 status and perturbed $\mathrm{pRb}$ cell cycle control. Oncogene 21: 1510-1517, 2002.

27. Califano J, van der Reit $\mathrm{P}$, Westra $\mathrm{W}$, et al: Genetic progression model for head and neck cancer: implications for field cancerization. Cancer Res 56: 2488-2492, 1996.

28. Goodwin EC and DiMaio D: Repression of human papillomavirus oncogenes in HeLa cervical carcinoma cells causes the orderly reactivation of dormant tumor suppressor pathways. Proc Natl Acad Sci USA 97: 12513-12518, 2000.

29. Hawley-Nelson P, Vousden KH, Hubbert NL, Lowy DR and Schiller JT: HPV16 E6 and E7 proteins cooperate to immortalize human foreskin keratinocytes. EMBO J 8: 3905-3910, 1989. 
30. Wells SI, Francis DA, Karpova AY, Dowhanick JJ, Benson JD and Howley PM: Papillomavirus E2 induces senescence in HPV-positive cells via pRB- and p21(CIP)- dependent pathways. EMBO J 19: 5762-5771, 2000

31. Butz K, Geisen C, Ullmann A, Spitkovsky D and Hoppe-Seyler F: Cellular responses of HPV-positive cancer cells to genotoxic anti- cancer agents: repression of E6/E7-oncogene expression and induction of apoptosis. Int J Cancer 68: 506-513, 1996.

32. Bristow RG, Benchimol S and Hill RP: The p53 gene as a modifier of intrinsic radiosensitivity: implications for radiotherapy. Radiother Oncol 40: 197-223, 1996.

33. Lindquist D, Romanitan M, Hammarstedt L, et al: Human papillomavirus is a favourable prognostic factor in tonsillar cancer and its oncogenic role is supported by the expression of E6 and E7. Mol Oncol 1: 350-355, 2007.

34. Lopez-Ocejo O, Viloria-Petit A, Bequet-Romero M, et al Oncogenes and tumor angiogenesis: the HPV-16 E6 oncoprotein activates the vascular endothelial growth factor (VEGF) gene promoter in a p53 independent manner. Oncogene 19: 4611-4620, 2000 .

35. Toussaint-Smith E, Donner DB and Roman A: Expression of human papillomavirus type 16 E6 and E7 oncoproteins in primary foreskin keratinocytes is sufficient to alter the expression of angiogenic factors. Oncogene 23: 2988-2995, 2004.

36. Tang X, Zhang Q, Nishitani J, et al: Overexpression of human papillomavirus type 16 oncoproteins enhances hypoxia-inducible factor 1 alpha protein accumulation and vascular endothelial growth factor expression in human cervical carcinoma cells. Clin Cancer Res 13: 2568-2576, 2007.

37. Dahm-Daphi J: p53: biology and role for cellular radiosensitivity. Strahlenther Onkol 176: 278-285, 2000

38. Lindel K, Beer KT, Laissue J, Greiner RH and Aebersold DM: Human papillomavirus positive squamous cell carcinoma of the oropharynx: a radiosensitive subgroup of head and neck carcinoma. Cancer 92: 805-813, 2001

39. De Wever O and Mareel M: Role of tissue stroma in cancer cell invasion. J Pathol 429-447, 2003.

40. Tlsty TD and Hein PW: Know their neighbor: stromal cells can contribute oncogenic signals. Curr Opin Genet Dev 11: 54-59, 2001.

41. Bhowmick NA, Neilson EG and Moses HL: Stromal fibroblast in cancer initiation and progression. Nature 432: 332-337, 2004.

42. Bhowmick NA, Chytil A, Plieth D, et al: TGFbeta signaling in fibroblasts modulates the oncogenic potential of adjacent epithelia. Science 303: 848-851,2004

43. Micke $\mathrm{P}$ and Ostman A: Exploring the tumor environment: cancer associated fibroblasts as targets in cancer therapy. Expert Opin Ther Targets 9: 1217-1233, 2005.

44. Joyce JA: Therapeutic targeting of the tumor microenvironment Cancer Cell 7: 513-520, 2005.

45. Hanahan D and Weinberg RA: The hallmarks of cancer. Cell 100: 57-70, 2000.

46. Ferrara N: The role of vascular endothelial growth factor in pathological angiogenesis. Breast Cancer Res Treat 36: 127-137, 1995.

47. Zhang HT, Craft P, Scott PA, et al: Enhancement of tumor growth and vascular density by transfection of vascular endothelial cell growth factor into MCF-7 human breast carcinoma cells. J Nat Cancer Inst 87: 213-219, 1995.

48. Eisma RJ, Spiro JD and Kreutzer DL: Vascular endothelial growth factor expression in head and neck squamous cell carcinoma. Am J Surg 174: 513-517, 1997.

49. Mineta $\mathrm{H}$, Miura $\mathrm{K}$ and Ogino T: Prognostic value of vascular endothelial growth factor in head and neck squamous cell carcinomas. Br J Cancer 83: 775-781, 2000.

50. Salven P, Heikkilä P and Anttonen A: Vascular endothelial growth factor in squamous cell head and neck carcinoma: expression and prognostic significance. Mod Pathol 10: 1128-1133, 1997.

51. Riedel F, Götte K and Schwalb J: Serum levels of vascular endothelial growth factor in patients with head and neck cancer. Eur Arch Otorhinolaryngol 257: 332-336, 2000.
52. Riedel F, Götte K and Schwalb J: Expression of vascular endothelial growth factor correlates with angiogenesis and p53 mutations in head and neck squamous cell carcinoma. Acta Otolaryngol 120: 105-111, 2000.

53. Le Buanec H, D'Anna R, Lachgar A, et al: HPV-16 E7 but not E6 oncogenic protein triggers both cellular immunosuppression and angiogenic processes. Biomed Pharmacother 53: 424-431, 1999.

54. Heldin $\mathrm{CH}$ and Westermark B: Mechanism of action and in vivo role of platelet-derived growth factor. Physiol Rev 79: 1283-1316, 1999.

55. Risau W, Drexler H, Mironov V, et al: Platelet-derived growth factor is angiogenic in vivo. Growth Factors 7: 261-266, 1992.

56. Pietras K, Ostman A, Sjöquist M, et al: Inhibition of plateletderived growth factor receptors reduces interstitial hypertension and increases transcapillary transport in tumors. Cancer Res 61: 2929-2934, 2001

57. Forsberg K, Valyi-Nagy I and Heldin $\mathrm{CH}$ : Platelet-derived growth factor (PDGF) in oncogenesis: development of a vascular connective tissue stroma in xenotransplanted human melanoma producing PDGF-BB. Proc Natl Acad Sci USA 90: 393-397, 1993.

58. Dong J, Grunstein J, Tejada M, et al: VEGF-null cells require PDGFR alpha signaling-mediated stromal fibroblast recruitment for tumorigenesis. EMBO J 23: 2800-2810, 2004.

59. Shao ZM, Nguyen M and Barsky SH: Human breast carcinoma desmoplasia is PDGF initiated. Oncogene 19: 4337-4345, 2000.

60. Skobe $M$ and Fusenig NE: Tumorigenic conversion of immortal human keratinocytes through stromal cell activation. Proc Natl Acad Sci USA 95: 1050-1055, 1998.

61. Bran B, Bran G and Hörmann K: The platelet-derived growth factor receptor as a target for vascular endothelial growth factormediated anti-angiogenic therapy in head and neck cancer. Int J Oncol 34: 255-261, 2009.

62. Blume-Jensen P and Hunter T: Oncogenic kinase signalling. Nature 411: 355-365, 2001.

63. Druker BJ and Lydon NB: Lessons learned from the development of an abl tyrosine-kinase inhibitor for chronic myelogenous leukemia. J Clin Invest 105: 3-7, 2000.

64. O'Dwyer ME, Mauro MJ and Druker BJ: STI571 as a targeted therapy for CML. Cancer Invest 21: 429-438, 2003.

65. Roskoski R Jr: STI-571: an anticancer protein-tyrosine kinase inhibitor. Biochem Biophys Res Commun 309: 709-717, 2003.

66. Shore SK, Bogart SL and Reddy EP: Activation of murine c-Abl protooncogene: effect of a point mutation on oncogenic activation. Proc Natl Acad Sci USA 87: 6502-6506, 1990.

67. Croom KF and Perry CM: Imatinib mesylate: in the treatment of gastrointestinal stromal tumors. Drugs 63: 513-522, 2003.

68. Demetri GD, von Mehren M, Blanke CD, et al: Efficacy and safety of imatinib mesylate in advanced gastrointestinal stromal tumors. N Engl J Med 347: 472-480, 2002.

69. Beppu K, Jaboine J, Merchant MS, et al: Effect of imatinib mesylate on neuroblastoma tumorigenesis and vascular endothelial growth factor expression. J Natl Cancer Inst 96: 46-55, 2004.

70. Vlahovic G, Ponce AM, Rabbani Z, et al: Treatment with imatinib improves drug delivery and efficacy in NSCLC xenografts. Br J Cancer 97: 735-740, 2007.

71. Wang-Rodriguez J, Lopez JP and Altuna X: STI-571 (Gleevec) Potentiates the effect of cisplatin in inhibiting the proliferation of head and neck squamous cell carcinoma in vitro. Laryngoscope 116: 1409-1416, 2006.

72. Schultz JD, Rotunno S, Sauter A, et al: Synergistic effects of imatinib and carboplatin on VEGF, PDGF and PDGF-R $\alpha / \beta$ expression in squamous cell carcinoma of the head and neck in vitro. Int J Oncol 38: 1001-1012, 2011.

73. Matei D, Kelich S, Cao L, et al: PDGF BB induces VEGF secretion in ovarian cancer. Cancer Biol Ther 6: 1951-1959, 2007. 